\title{
Flexural Behaviour of HYFRC Beam Reinforced with GFRP Rebar
}

\author{
S.Kamala Kannan
}

\begin{abstract}
This paper offers the experimental have a take a look at at the flexural behaviour of HYFRC beams bolstered with glass fiber bolstered polymer (GFRP) rebar and in comparison with everyday metal reinforcement beams. Three beams reinforced with GFRP rebar and three beams of traditional concrete metal strengthened with absolutely six beams have been casted and tested under two points loading. The partner specimens were casted along with beam and tested for concrete homes. Steel and glass fibres are used toimprove the concrete assets. From checking out, load carrying capability, loaddeflection traits, crack sample, crack width, concrete traces throughout move phase and failure mode have been mentioned stiffness, ductility and power dissipation potential had been additionally calculated. The average ultimate load wearing potential of GFRP rebar and normal steel reinforcement beam is one hundred twenty five. $8 K N$ and ninety seven.5KN respectively. The most deflection cited at their closing load inside the GFRP rebar and regular metal reinforcement beam is 27. Three mm and sixteen. $3 \mathrm{~mm}$ respectively. It changed into also found that after load elimination, deflected GFRP beam regain its authentic function and crack width also reduced. In metal beam, metallic rebar were yielded, after load elimination, no deflection regain and crack width reduction have been observed.
\end{abstract}

Keywords- GFRP, Hybrid fibre, flexural testing, stiffness, ductility and energy dissipation capacity.

\section{INTRODUCTION}

Concrete is a versatile construction material used global. Concrete technologists are constantly carrying out the research to beautify the overall performance of concrete to satisfy the beneficial, energy and sturdiness requirement. Concrete has the downside of being weak in anxiety, porous and prone for environmental attack. The problems of simple concrete were overcome is happy, via including fibre to decorate density for better overall performance. The necessity for new non corrosive material because of corrosion problems associated with metal.

Glass fibre strengthened polymer (GFRP) bars and hybrid fibre had been applied in present studies to deal with the problem of corrosion related to metal. GFRP rebar are mild in weight and approximately one $1 /$ three in evaluation to that metallic.

In the winning paintings was to investigate loaddeformation tendencies, load wearing capacity, failure mode, stress-strain traits at some point of pass segment and flexural universal performance of HYFRC beam strengthened with GFRP rebar and it modified into compared the flexural behaviour of conventional concrete beam strengthened with rebar.

Annadurai et al[1]have experimentally studied the flexural behaviour of excessive energy concrete grade of M60 the use of hooked ends metallic fibres, polyolefin

Revised Manuscript Received on July 18, 2019.

Dr.S.KamalaKannan, Associate Professor \& Head of the Department, Department of civil Engineering, IES College of Engineering. Kerala, India immediately fibres in numerous extent fractions. In this study durability indices were calculated through using the experimental load deflection. They had been centered on comparing the ductility and power absorption ability. The check consequences confirmed that hybrid fibre of quantity fraction 2\% with metal eighty\%-polyolefin $20 \%$ combination specimen improves the flexural performance notably compared with that of manipulate specimen and steel fibre bolstered immoderate strength concrete specimen. PriyankaDilip et al[2] they have got described the look at on the mechanical general performance of Hybrid fibre bolstered concrete (HFRC). The addition of small carefully spaced and uniformly dispersed fibres to concrete might act as crack arrester and may considerably enhance its static and dynamic residences. Here Steel fibre and polyolefin fibre are used as Hybrid fibre. They are used in 4 distinct proportions as $0 \%, 0$. Five $\%, 1 \%, 1$. Five $\%$ and a pair of $\%$ in this examine. They concluded that fibre content material in concrete increases, the energy additionally will increase as much as a sure quantity. Mix containing $1 \%$ Hybrid fiber confirmed most overall performance. Yaminiroja, et al [3] have investigatedstaticbehavior Of concrete beams bolstered with GFRP beams have been carried out to have a look at the flexural behavior underneath static monotonic loading. Due to the low modulus of elasticity of GFRP bars, the crack initiation load turned into determined to be early in beams with GFRP reinforcement even as in comparison to beams with traditional TMT reinforcement. They concluded that not unusual values of crack initiation loads for beams with GFRP and TMT reinforcement had been eleven. Four $\mathrm{kN}$ and $20.1 \mathrm{kN}$ respectively. Similarly, the common values of closing load sporting capacity for beams with GFRP and TMT reinforcement have been 80 two.Nine $\mathrm{kN}$ and $97.6 \mathrm{kN}$ respectively

\section{EXPERIMENTAL PROGRAM}

\subsection{MATERIALS USED}

\subsubsection{CEMENT:}

Ordinary Portland cement conforms to IS 10262-2009 penna cement 53 grade produced from single source was used. The specific gravity of the cement is 3.15.

\subsubsection{FINE AGGREGATE:}

Locally available river sandwas used as fine aggregate which passes through $4.75 \mathrm{~mm}$ as per IS $383-1978$. The specific gravity of the fine aggregate is 2.67. Zone 3 was used. The fineness modulus of aggregate was 2.8 .

Published By:

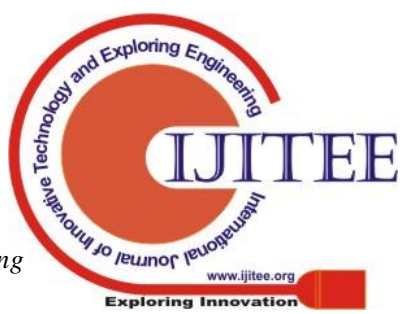




\subsubsection{COARSE AGGREGATE:}

Locally available coarse aggregate brought from hosur $20 \mathrm{~mm}$ size aggregate was used. The specific gravity of the coarse aggregate is 2.74.The fineness modulus of aggregate was 3.58 .

\subsubsection{WATER:}

Potable water which is available in laboratory is used for casting and curing of specimen as per IS 456-2000.W/C .45 was in mix.

\subsubsection{STEEL FIBER:}

Steelfibre with hooked end was used. The properties of steel fibre with their specification are mentioned below table 1.

TABLE 1:Properties of Steel Fibre

\begin{tabular}{|c|c|}
\hline PROPERTIES & SPECIFICATIONS \\
\hline Type of steel fibre & Crimped \\
\hline Material & Low carbon drawn flat wire \\
\hline Length & $50 \mathrm{~mm}$ \\
\hline Diameter of fibre & $0.5 \mathrm{~mm}$ \\
\hline Aspect ratio & 50 \\
\hline Percentage & $1 \%$ (volume of cement) \\
\hline
\end{tabular}

\subsubsection{GLASS FIBER:}

CEMFIL anti crack AR glass fibre(alkali resistant) was used. The properties of glass fibre with the specification are mentioned below table 2 .

TABLE 2:Properties of Glass Fibre

\begin{tabular}{|c|c|}
\hline PROPERTIES & SPECIFICATIONS \\
\hline Type of glass fibre & Alkali resistant (AR) \\
\hline Length & $12 \mathrm{~mm}$ \\
\hline Diameter of fibre & 14 micron \\
\hline Aspect ratio & 857.1 \\
\hline percentage & $1 \%$ (volume of cement) \\
\hline
\end{tabular}

\subsubsection{GFRP REBAR:}

In the existing studies substitute of metallic reinforcement as glass fiber bolstered polymer. GFRP bars posse's mechanical houses special from metal bars, inclusive of high tensile energy mixed with low elastic modulus and elastic brittle pressure-pressure relationship.

\subsubsection{CONCRETEMIX PROPOTION:}

In the present studies substitute of steel reinforcement as glass fiber strengthened polymer. GFRP bars posse's mechanical houses unique from metal bars, such as high tensile energy mixed with low elastic modulus and elastic brittle stress-strain relationship.

\subsubsection{CONCRETEMIX PROPOTION:}

Mix share of M35 grade concrete end up designed as in keeping with IS 10262-2009 and IS 456-2000. The proportion and w/c ratio for M35 is 1:1.Seventy seven:2.84, 0.45 .

\subsection{REINFORCEMENT DETAILS:}

The experimental research includes casting and testing of six beams of dimension (1800 mm duration, 100 fifty $\mathrm{mm}$ width and $250 \mathrm{~mm}$ intensity). Beams had been absolutely supported at their ends with the effective span of $1500 \mathrm{~mm}$.
A view of longitudinal section and go segment of a wellknown beam specimen is confirmed fig.1.Three beams have been casted with HYFRC with GFRP rebar as longitudinal reinforcement. 3 beams were casted with conventional concrete with steel rebar in HYFRC beam steel and glass fibres were used. GFRP 2nos of rebar of $10 \mathrm{~mm}$ diameter was used as reinforcement at top and bottom for shear $6 \mathrm{~mm}$ stirrups $6 \mathrm{~mm}$ diameter 2 legged vertical were used at $150 \mathrm{~mm}$ c/c. Steel 2 nos of $10 \mathrm{~mm}$ diameter main bar and $\mathrm{mm}$ stirrups were used for 3 beams. TMT $10 \mathrm{~mm}$ diameter main bar and six mm stirrups were used for 3 beams. Reinforcement details shown below Fig 1 .

\section{REINFORCEMENTDETAILS}

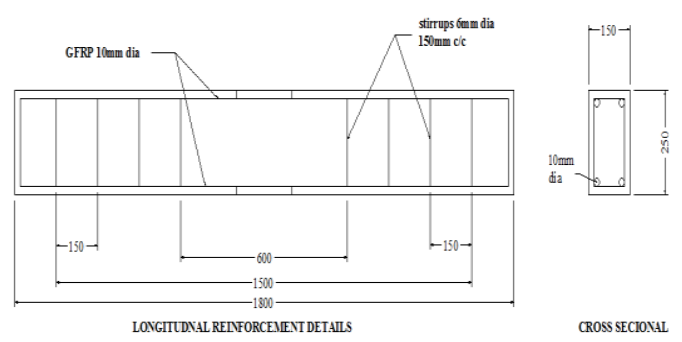

Figure1Reinforcement Details

\subsection{CASTING AND CURING}

Compressive strength of HYFRC companion specimen was casted as follows. 3nos of 150X150X150 mm cube, 150x300 mm cylinders and 3 nos of prism were casted 100x $100 \times 500 \mathrm{~mm}$ prism specimen were casted and tested for compressive, split tensile and flexural strength of concrete. Similarly for conventional concrete above side specimens and tested. Specimens are shown below Fig $2 \mathrm{a}, \mathrm{b}, \mathrm{c}$

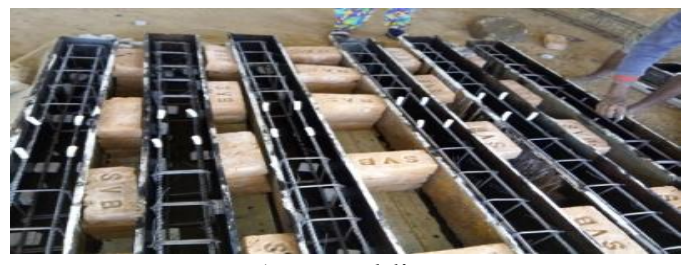

a) moulding

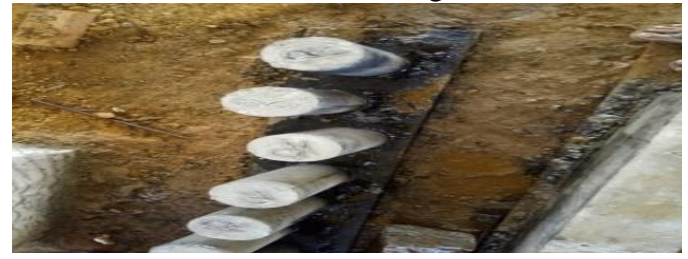

b) casting

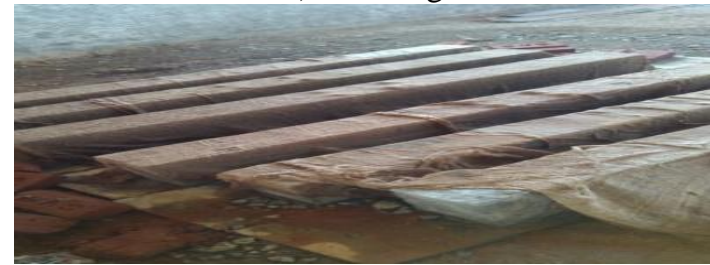

c) curing

Figure2moulding,Casting and Curing of Specimens

Blue Eyes Intelligence Engineering

\& Sciences Publication

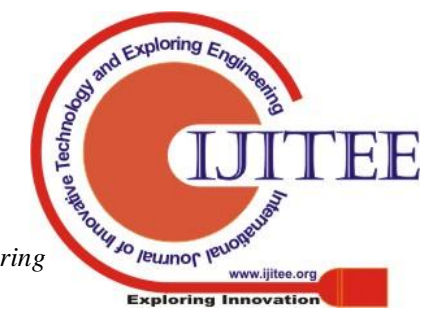




\section{TESTPROGRAMME}

The test setup involves a two point loading system by using a spread beam and two rollers. Totally 3 LVDTs one $100 \mathrm{~mm}$, two 50mm LVDTs were used to measure deflection placed at the mid span of beam along the tension side. Two $50 \mathrm{~mm}$ LVTDs were used under two points loading to measure deflection. A $50 \mathrm{~mm}$ dial cage was placed near beam end to measure the rotation. Pellets were placed as shown in Fig 3 at mid span across cross section of beam to measure concrete strain. The point loads acts at a distance of $200 \mathrm{~mm}$ from the mid span along the compression side of the beam. Test setup shown in Fig 4 .

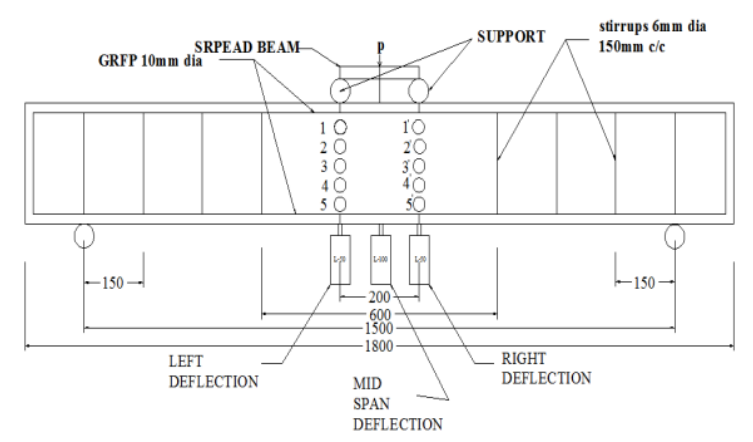

Figure 3 Placing of LVDTs

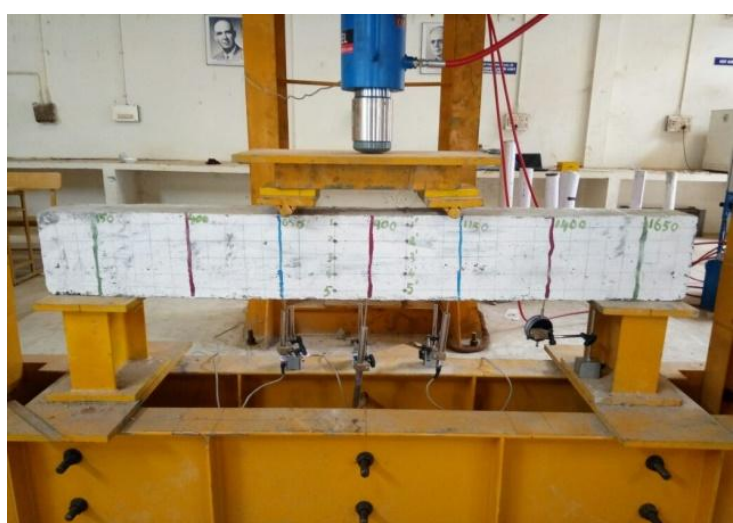

Figure 4Test setup

\section{RESULT AND DISCUSSION}

\subsection{CUBE TEST RESULT}

Compressive electricity Is the maximum compressive pressure that, beneath a regularly applied load, given strong cloth can preserve without fracture. Compressive electricity is calculated by dividing the maximum load through the authentic move section area of the specimen in compression take a look at. Cube check result referred to beneath in table 3.

TABLE 3:Test Results of Compressive Strength of Concrete

\begin{tabular}{|c|c|c|c|c|c|c|c|}
\hline S.NO & SPECIMEN & $\begin{array}{l}\text { WEIGHT } \\
(\mathrm{kg})\end{array}$ & $\begin{array}{l}\text { DIMENSION } \\
(\mathrm{mm})\end{array}$ & $\begin{array}{l}\text { INITIAL } \\
\text { LOAD (kN) }\end{array}$ & $\begin{array}{c}\text { FINAL } \\
\text { LOAD } \\
(\mathrm{kN})\end{array}$ & $\begin{array}{c}\text { COMPRESSIVE } \\
\text { STRENGTH }\left(\mathrm{N} / \mathrm{mm}^{2}\right)\end{array}$ & $\begin{array}{l}\mathrm{AVG} \\
\left(\mathrm{N} / \mathrm{mm}^{2}\right)\end{array}$ \\
\hline 1. & HYFRC-1 & 7.870 & $150 \times 150$ & 898 & 944 & 41.95 & \multirow{3}{*}{40.66} \\
\hline 2. & HYFRC-2 & 7.945 & $150 \times 150$ & 720 & 880 & 39.11 & \\
\hline 3. & HYFRC-3 & 7.475 & $150 \times 150$ & 760 & 921 & 40.93 & \\
\hline 4. & $\mathrm{~N}-1$ & 8.645 & $150 \times 150$ & 763 & 874 & 38.48 & \multirow{3}{*}{38.37} \\
\hline 5. & $\mathrm{~N}-2$ & 8.415 & $150 \times 150$ & 516 & 824 & 38.07 & \\
\hline 6. & $\mathrm{~N}-3$ & 8.515 & $150 \times 150$ & 652 & 839 & 38.57 & \\
\hline
\end{tabular}

\subsection{CYLINDER TEST RESULT}

The tensile energy of concrete is not able to degree without delay. Splitting tensile power Test on concrete cylinder is a way to determine the tensile strength of concrete. Concrete develops cracks when subjected to tensile forces. Thus, it's miles vital to determine the tensile strength of concrete to determine the load at which the concrete individuals can also moreover crack. Cylinder test results are referred to below in desk 4.

TABLE 4:Test Result of Split Tensile Strength of Concrete

\begin{tabular}{|c|c|c|c|c|c|c|}
\hline S.NO & SPECIMEN & $\begin{array}{l}\text { WEIGHT } \\
(\mathrm{kg})\end{array}$ & $\begin{array}{l}\text { DIMENSION } \\
(\mathrm{mm})\end{array}$ & $\begin{array}{c}\text { FINAL } \\
\text { LOAD } \\
(\mathrm{kN})\end{array}$ & $\begin{array}{c}\text { SPLIT TENSILE } \\
\text { STRENGTH }\left(\mathrm{N} / \mathrm{mm}^{2}\right)\end{array}$ & $\begin{array}{c}\mathrm{AVG} \\
\left(\mathrm{N} / \mathrm{mm}^{2}\right)\end{array}$ \\
\hline 1. & HYFRC-1 & 12.825 & $150 \times 300$ & 189 & 2.7 & \multirow{3}{*}{2.87} \\
\hline 2. & HYFRC-2 & 13.030 & $150 \times 300$ & 194 & 2.9 & \\
\hline 3. & HYFRC-3 & 13.345 & $150 \times 300$ & 202 & 3.01 & \\
\hline 4. & $\mathrm{~N}-1$ & 13.175 & $150 \times 300$ & 187 & 2.7 & \multirow{3}{*}{2.63} \\
\hline 5. & $\mathrm{~N}-2$ & 13.080 & $150 \times 300$ & 194 & 2.74 & \\
\hline 6. & $\mathrm{~N}-3$ & 13.155 & $150 \times 300$ & 173 & 2.45 & \\
\hline
\end{tabular}

\subsection{PRISM TEST RESULT}

Flexural energy additionally called modulus of rupture, or bend energy, or transverse rupture is a fabric belongings, defined as the pressure in a fabric simply before it yields in a flexure take a look at. 
FLEXURAL BEHAVIOUR OF HYFRC BEAM REINFORCED WITH GFRP REBAR

TABLE 5:Test Result of Flexural Strength of Concrete

\begin{tabular}{|c|c|c|c|c|c|}
\hline S.NO & SPECIMEN & WEIGHT (kg) & DIMENSION(mm) & FINAL LOAD(kN) & FLXERUAL STRENGTH(N/mm²) \\
\hline 1. & HYFRC-1 & 13.125 & $100 \times 100 \times 500$ & 14.5 & \multirow{3}{*}{12.83} \\
\hline 2. & HYFRC-2 & 13.330 & $100 \times 100 \times 500$ & 12 & \\
\hline 3. & HYFRC-3 & 13.145 & $100 \times 100 \times 500$ & 12 & \\
\hline 4. & $\mathrm{~N}-1$ & 12.815 & $100 \times 100 \times 500$ & 9.25 & \multirow{3}{*}{8.75} \\
\hline 5. & $\mathrm{~N}-2$ & 13.200 & $100 \times 100 \times 500$ & 10.5 & \\
\hline 6. & $\mathrm{~N}-3$ & 12.7 & $100 \times 100 \times 500$ & 6.5 & \\
\hline
\end{tabular}

\subsection{LOAD CARRYING CAPACITY}

A beam is a structural detail that typically resists loads carried out laterally to the beam's axis. Its mode of deflection is normally by way of way of bending.

TABLE 6:Test Result of load carrying capacity

\begin{tabular}{|l|l|l|l|}
\hline S.No & SPECIMEN & $\begin{array}{l}\text { INITIAL } \\
\text { CRACK } \\
\text { LOAD }(\mathrm{kN})\end{array}$ & $\begin{array}{l}\text { ULTIMATE } \\
\text { LOAD }(\mathrm{kN})\end{array}$ \\
\hline 1. & HYFRC-1 & 40 & 160.3 \\
\hline 2. & HYFRC-2 & 30 & 110.1 \\
\hline 3. & HYFRC-3 & 35 & 107 \\
\hline 4. & $\mathrm{~N}-1$ & 21 & 76.6 \\
\hline 5. & $\mathrm{~N}-2$ & 20.6 & 120 \\
\hline 6. & $\mathrm{~N}-3$ & 30 & 96 \\
\hline
\end{tabular}

LOAD CARRYING CAPACITY OF HYFRC BEAM REINFORCED WITH GFRP REBAR

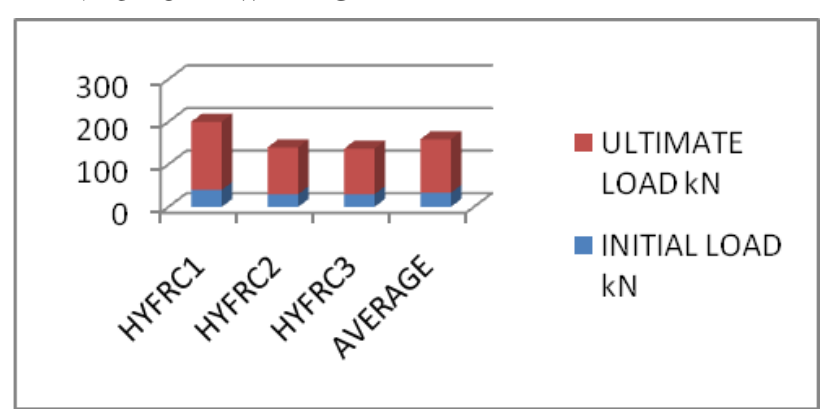

Figure 5 Load Carrying Capacity ofHyfrc Beam

LOAD CARRYING CAPACITYOF CONVENTIONAL BEAM REINFORCED WITH STEEL BAR

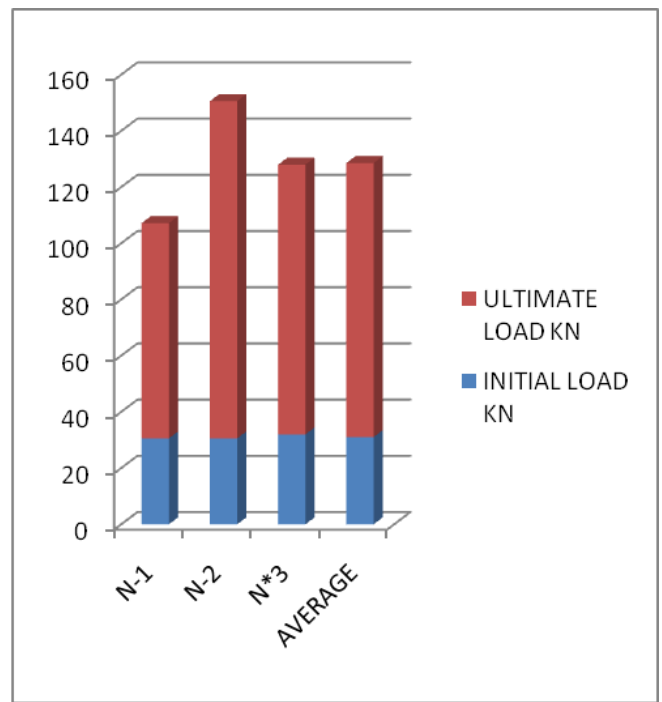

Figure 6Load Carrying Capacity of Normal Beam

\subsection{LOADVERSUS DEFLECTIONCHARACTERISTICS}

In this 3 LVDTs were placed to measure the left deflection, middle deflection and right deflection (such as deflection 1, deflection 2 and deflection 3).

FOR SPECIMEN 1

TABLE 7:Various Deflection of HYFRC-1

\begin{tabular}{|c|c|c|c|c|}
\hline S.NO & LOAD $(\mathrm{kN})$ & DEFLECTION1 & DEFLECTION2 & DEFLECTION3 \\
\hline 1. & 0 & 0 & 0 & 0 \\
\hline 2. & 25 & 0.7 & 0.7 & 0.8 \\
\hline 3. & 38 & 3.7 & 3.9 & 3.9 \\
\hline 4. & 48 & 5.7 & 5.9 & 5.9 \\
\hline 5. & 60 & 7.6 & 8.1 & 7.8 \\
\hline 6. & 73 & 10.1 & 10.7 & 10.1 \\
\hline 7. & 83 & 11 & 11.5 & 11 \\
\hline 8. & 93 & 13.2 & 14.1 & 13.4 \\
\hline 9. & 103 & 15.1 & 16.1 & 15.4 \\
\hline 10. & 113 & 16.7 & 17.6 & 17.3 \\
\hline 11. & 122 & 17.6 & 18.7 & 18.2 \\
\hline 12. & 135 & 20.1 & 21.5 & 21 \\
\hline 13. & 143 & 21.5 & 22.6 & 22.3 \\
\hline 14. & 153 & 25.3 & 26.1 & 25.8 \\
\hline 15. & 160.3 & 28.9 & 29.7 & 29 \\
\hline
\end{tabular}




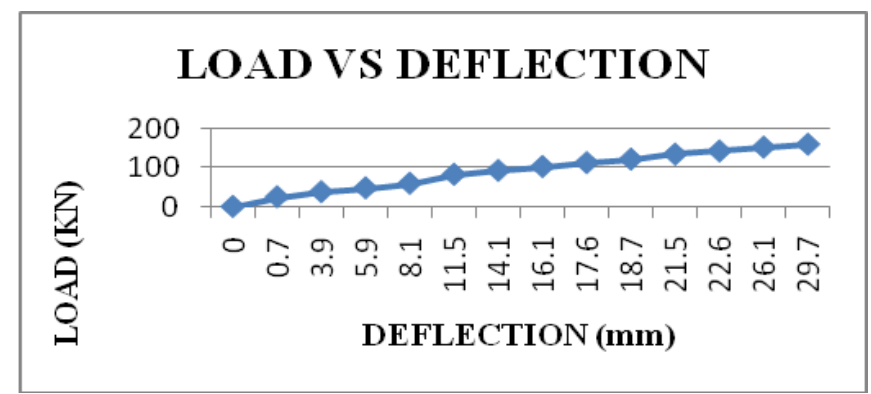

Figure 7 Load Vs Middle Deflection Hyfrc - 1

FOR SPECIMEN 2

TABLE 8: Various Deflection of HYFRC-2

\begin{tabular}{|c|c|c|c|c|}
\hline S.NO & LOAD $(\mathrm{kN})$ & DEFLECTION1 & DEFLECTION2 & DEFLECTION3 \\
\hline 1. & 0 & 0 & 0 & 0 \\
\hline 2. & 10 & 0.1 & 0.1 & 0.1 \\
\hline 3. & 20 & 0.2 & 0.3 & 0.2 \\
\hline 4. & 30 & 0.8 & 0.8 & 0.8 \\
\hline 5. & 40 & 3.8 & 4.4 & 3.9 \\
\hline 6. & 50 & 6.7 & 7.1 & 6.2 \\
\hline 7. & 60 & 9.5 & 9.8 & 8.8 \\
\hline 8. & 70 & 11.4 & 11.7 & 10.2 \\
\hline 9. & 80 & 14 & 14.2 & 12.3 \\
\hline 10. & 90 & 16.2 & 16.3 & 17.1 \\
\hline 11. & 100 & 19.9 & 20.5 & 20.7 \\
\hline 12. & 110.3 & 24.1 & 25.3 & \\
\hline
\end{tabular}

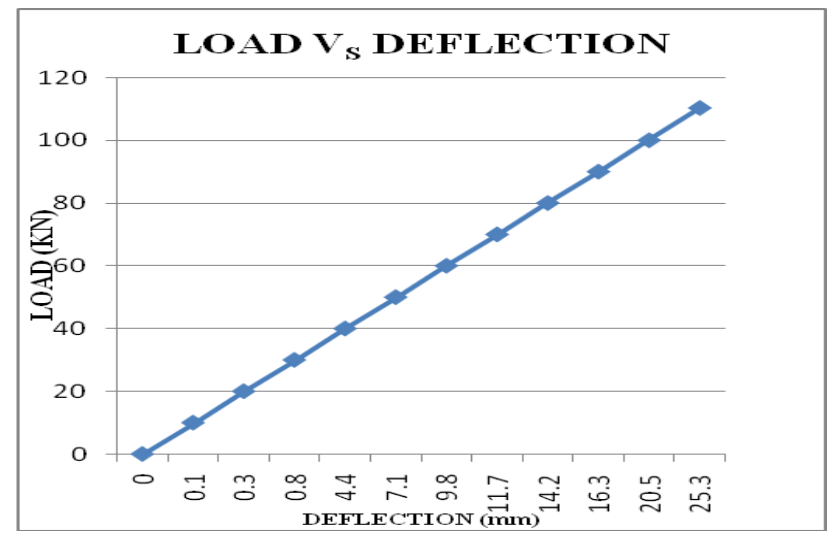

Figure 8 LoadVs Middle Deflection Hyfrc - 2

FOR SPECIMEN 3:

TABLE 9: Various Deflection of HYFRC-3

\begin{tabular}{|c|c|c|c|c|}
\hline S.NO & LOAD $(\mathrm{kN})$ & DEFLECTION1 $\mathrm{mm}$ & DEFLECTION2 $\mathrm{mm}$ & DEFLECTION3 mm \\
\hline 1. & 0 & 0 & 0 & 0 \\
\hline 2. & 10 & 0.2 & 0.1 & 0.2 \\
\hline 3. & 25 & 1.1 & 1.1 & 1.1 \\
\hline 4. & 35 & 5.9 & 6.3 & 5.9 \\
\hline 5. & 45 & 9 & 9.6 & 9.7 \\
\hline 6. & 60 & 9.1 & 10 & 11.6 \\
\hline 7. & 70 & 11.6 & 13 & 14.4 \\
\hline 8. & 85 & 14.4 & 16.2 & 19 \\
\hline 9. & 100 & 19 & 22 & 24 \\
\hline 10. & 107 & 24 & 27 & \\
\hline
\end{tabular}


FLEXURAL BEHAVIOUR OF HYFRC BEAM REINFORCED WITH GFRP REBAR

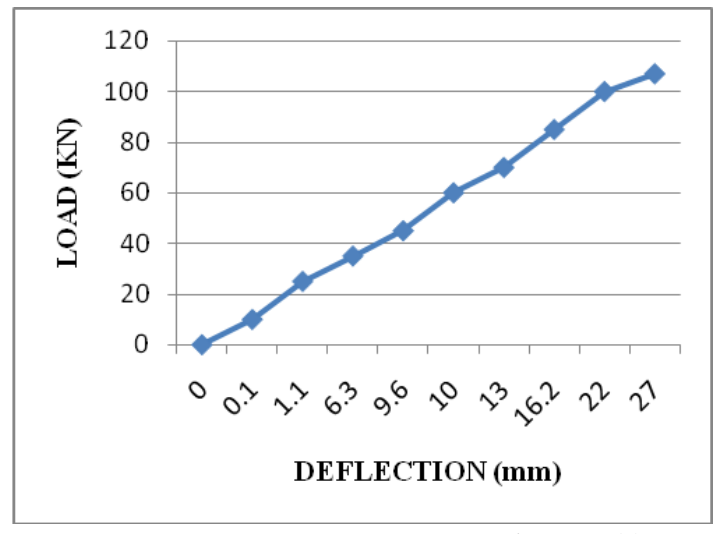

Figure 9 Load Vs Middle Deflection Hyfre - 3

CONCRETE BEAM REINFORCED WITH STEEL REBAR

FOR SPECIMEN 1

TABLE 10:Various Deflection of NORMAL -1

\begin{tabular}{|c|c|c|c|c|}
\hline S.NO & LOAD $(\mathrm{kN})$ & DEFLECTION 1 $(\mathrm{mm})$ & DEFLECTION 2 $(\mathrm{mm})$ & DEFLECTION 3 $(\mathrm{mm})$ \\
\hline 1. & 0 & 0 & 0 & 0 \\
\hline 2. & 30.6 & 0 & 1.2 & 0.4 \\
\hline 3. & 40.6 & 0 & 1.3 & 0.4 \\
\hline 4. & 53.8 & 0.3 & 2.9 & 1.1 \\
\hline 5. & 57.2 & 0.5 & 3.4 & 1.4 \\
\hline 6. & 67 & 0.8 & 4.1 & 1.7 \\
\hline 7. & 76.6 & 2.8 & 8 & 3.6 \\
\hline
\end{tabular}

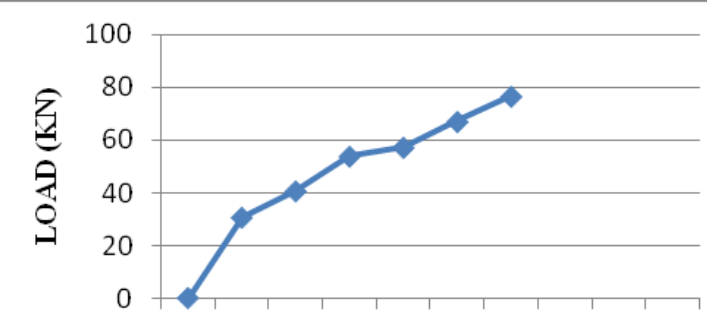

$\begin{array}{lllllll}0 & 1.2 & 1.3 & 2.9 & 3.4 & 4.1 & 8\end{array}$

DEFLECTION (nm)

Figure 9 Load Vs Middle Deflection Normal - 1

FOR SPECIMEN 2

TABLE 11: Various Deflections of Normal-2

\begin{tabular}{|c|c|c|c|c|}
\hline S.NO & LOAD $(\mathrm{kN})$ & DEFLECTION 1 $(\mathrm{mm})$ & DEFLECTION 2 $(\mathrm{mm})$ & DEFLECTION 3 $(\mathrm{mm})$ \\
\hline 1. & 0 & 0 & 0 & 0 \\
\hline 2. & 20.6 & 0.9 & 0.9 & 0.8 \\
\hline 3. & 30.6 & 1.4 & 1.8 & 1.3 \\
\hline 4. & 44 & 2.3 & 2.8 & 2.3 \\
\hline 5. & 54 & 3.4 & 3.9 & 3.4 \\
\hline 6. & 64.5 & 3.7 & 4.4 & 3.8 \\
\hline 7. & 76 & 4.3 & 5.1 & 4.5 \\
\hline 8. & 86 & 5.3 & 6.3 & 5.8 \\
\hline 9. & 120 & 14.8 & 17.1 & 17.6 \\
\hline
\end{tabular}




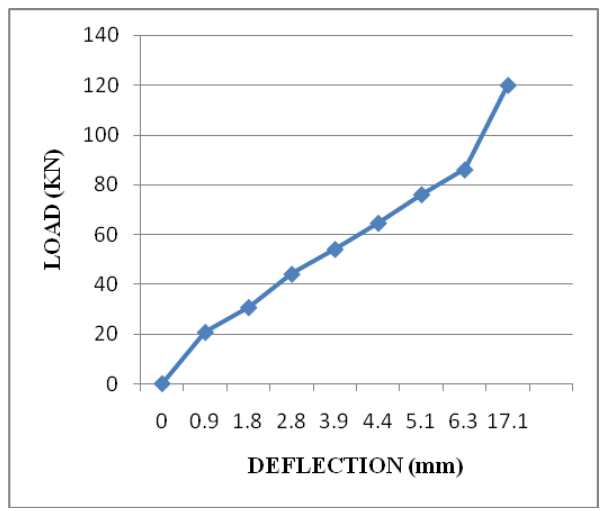

Figure 10 Load Vs Middle Deflection Normal - 2 FOR SPECIMEN 3

TABLE 12:Various Deflections of NORMAL -3

\begin{tabular}{|c|c|c|c|c|}
\hline S.NO & LOAD $(\mathrm{kN})$ & DEFLECTION 1 $(\mathrm{mm})$ & DEFLECTION 2 $(\mathrm{mm})$ & DEFLECTION 3 $(\mathrm{mm})$ \\
\hline 1. & 0 & 0 & 0 & 0 \\
\hline 2. & 21 & 0.3 & 1.0 & 1.3 \\
\hline 3. & 32 & 1.5 & 2 & 2.6 \\
\hline 4. & 42 & 2.2 & 3 & 3.4 \\
\hline 5. & 52 & 2.5 & 3 & 3.7 \\
\hline 6. & 63 & 3.4 & 4 & 4.8 \\
\hline 7. & 73 & 5.3 & 7 & 7 \\
\hline 8. & 96 & 22.1 & 24 & 23.9 \\
\hline
\end{tabular}

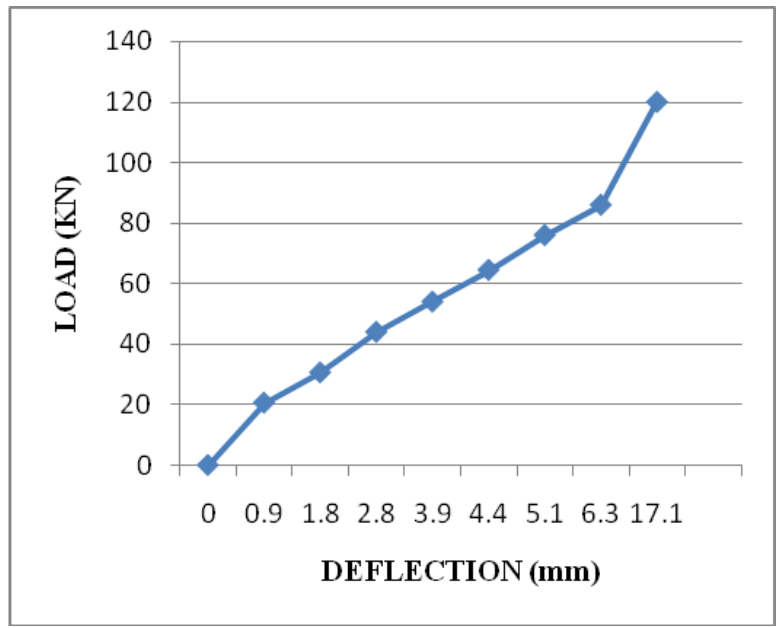

Figure 11 Load Vs Middle Deflection Normal - 3

\subsection{MOMENTVSROTATIONCHARACTERISTICS:}

It is the best second the beam can experience proper before or right as it starts offevolved to fail on paper. It is a mathematically determined range. It does not bear in mind, element of safety, nor inferior high-quality of cloth, human errors at some stage in fabrication or any other real world scenario. It's just a mathematical calculation of the beams capability in a perfect world.

The theoretical, applied bending moment that will cause failure in a reinforced concrete member through yield in the tensile reinforcing steel or crushing of concrete

\section{FOR BEAM SPECIMEN 1:}

TABLE 13:Ultimate Moment forHYFRC-1

\begin{tabular}{|c|c|c|c|}
\hline S.NO & $\begin{array}{c}\text { LOAD } \\
(\mathrm{kN})\end{array}$ & $\begin{array}{c}\mathrm{M} \\
(\mathrm{kN}-\mathrm{m})\end{array}$ & ROTATION \\
\hline 1. & 0 & 0 & 0 \\
\hline 2. & 25 & 16.25 & 0.05 \\
\hline 3. & 38 & 24.70 & 0.10 \\
\hline
\end{tabular}

\begin{tabular}{|c|c|c|c|}
\hline 4. & 48 & 31.20 & 0.15 \\
\hline 5. & 60 & 39.00 & 0.15 \\
\hline 6. & 73 & 47.45 & 0.20 \\
\hline 7. & 83 & 53.95 & 0.20 \\
\hline 8. & 93 & 60.45 & 0.25 \\
\hline 9. & 103 & 66.95 & 0.25 \\
\hline 10. & 113 & 73.45 & 0.25 \\
\hline 11. & 122 & 79.30 & 0.30 \\
\hline 12. & 135 & 87.75 & 0.35 \\
\hline 13. & 143 & 92.95 & 0.35 \\
\hline 14. & 153 & 99.45 & 0.45 \\
\hline 15. & 160.3 & 104.19 & 0.45 \\
\hline
\end{tabular}

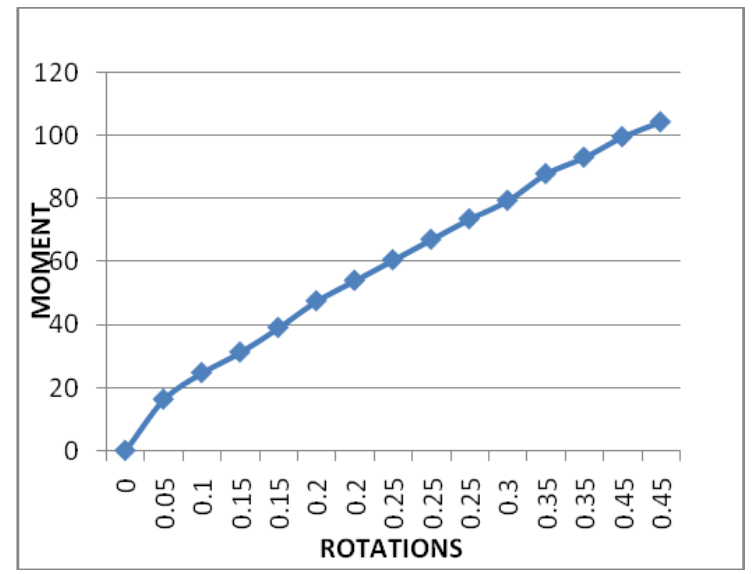

Figure 12 Moment Vs Rotation Hyfrc - 1

FOR BEAM SPECIMEN 2: 
TABLE 14:Ultimate Moment forHYFRC-2

\begin{tabular}{|c|c|c|c|}
\hline S.NO & $\begin{array}{c}\text { LOAD } \\
(\mathrm{kN})\end{array}$ & M (kN-m) & ROTATION \\
\hline 1. & 0 & 0 & 0 \\
\hline 2. & 10 & 6.5 & 0.0005 \\
\hline 3. & 20 & 13 & 0.0008 \\
\hline 4. & 30 & 19.5 & 0.050 \\
\hline 5. & 40 & 26 & 0.100 \\
\hline 6. & 50 & 32.5 & 0.100 \\
\hline 7. & 60 & 39 & 0.150 \\
\hline 8. & 70 & 45.5 & 0.150 \\
\hline 9. & 80 & 52 & 0.200 \\
\hline 10. & 90 & 58.5 & 0.200 \\
\hline 11. & 100 & 65 & 0.200 \\
\hline 12. & 110.3 & 71.69 & 0.250 \\
\hline
\end{tabular}

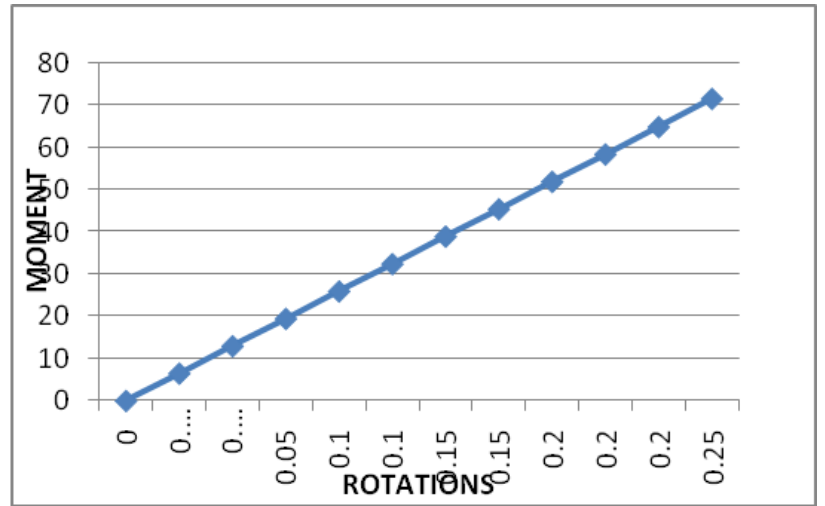

Figure 13 Moment Vs Rotation Hyfrc - 2

FOR SPECIMEN 3 :

TABLE 15:Ultimate Moment forHYFRC-3

\begin{tabular}{|c|c|c|c|}
\hline S.NO & LOAD $(\mathrm{kN})$ & M $(\mathrm{kN}-\mathrm{m})$ & ROTATION \\
\hline 1. & 0 & 0 & 0 \\
\hline 2. & 10 & 6.5 & 0.0002 \\
\hline 3. & 25 & 16.25 & 0.0504 \\
\hline 4. & 35 & 22.75 & 0.150 \\
\hline 5. & 45 & 29.25 & 0.150 \\
\hline 6. & 60 & 39 & 0.200 \\
\hline 7. & 70 & 45.5 & 0.200 \\
\hline 8. & 85 & 55.25 & 0.200 \\
\hline 9. & 100 & 65 & 0.250 \\
\hline 10. & 107 & 69.55 & 0.250 \\
\hline
\end{tabular}

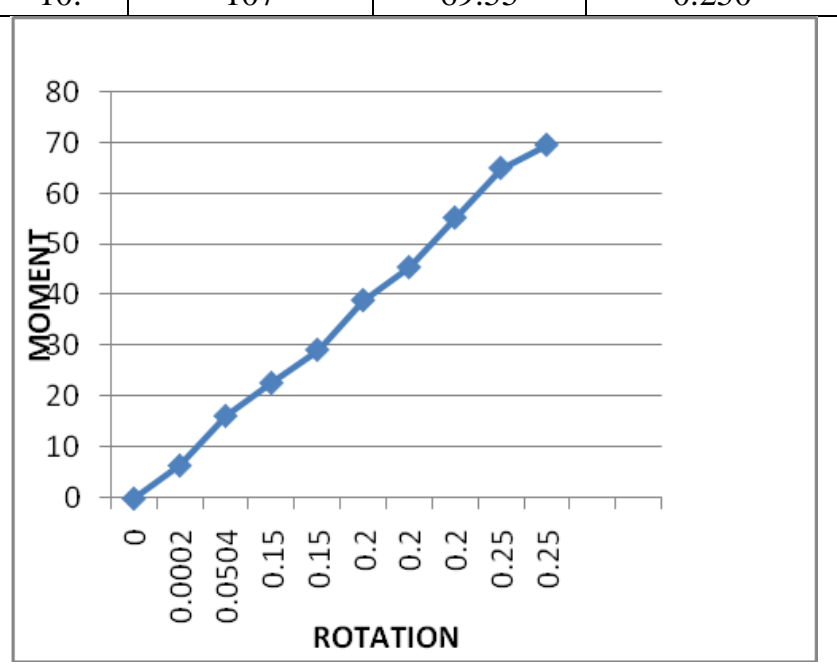

Figure 14 Moment Vs Rotation Hyfrc - 3
CONCRETE BEAM REINFORCED WITH STEEL REBAR

FOR SPECIMEN 1

TABLE 16:Ultimate Moments forNORMAL-1

\begin{tabular}{|c|c|c|c|}
\hline S.NO & LOAD $(\mathrm{kN})$ & M $(\mathrm{kN}-\mathrm{m})$ & ROTATION \\
\hline 1. & 0 & 0 & 0 \\
\hline 2. & 30.6 & 19.89 & 0.05 \\
\hline 3. & 40.6 & 26.39 & 0.05 \\
\hline 4. & 53.8 & 34.97 & 0.10 \\
\hline 5. & 57.2 & 37.18 & 0.10 \\
\hline 6. & 67 & 43.55 & 0.10 \\
\hline 7. & 76.6 & 49.79 & 0.15 \\
\hline
\end{tabular}

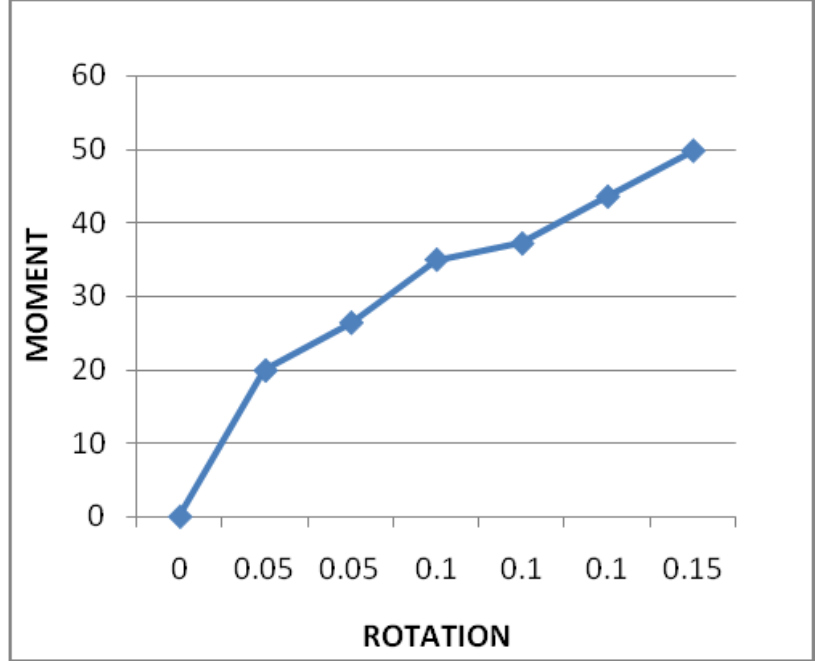

Figure 15 Moment Vs Rotation Normal - 1

FOR SPECIMEN 2:

TABLE 17:Ultimate Moments forNORMAL-2

\begin{tabular}{|c|c|c|c|}
\hline S.NO & LOAD $(\mathrm{kN})$ & M $(\mathrm{kN}-\mathrm{m})$ & ROTATION \\
\hline 1. & 0 & 0 & 0 \\
\hline 2. & 20.6 & 13.39 & 0 \\
\hline 3. & 30.6 & 19.89 & 0 \\
\hline 4. & 44 & 28.60 & 0.05 \\
\hline 5. & 54 & 35.10 & 0.05 \\
\hline 6. & 64.5 & 41.92 & 0.05 \\
\hline 7. & 76 & 49.40 & 0.05 \\
\hline 8. & 86 & 55.90 & 0.10 \\
\hline 9. & 120 & 78.0 & 0.20 \\
\hline
\end{tabular}

Published By: 


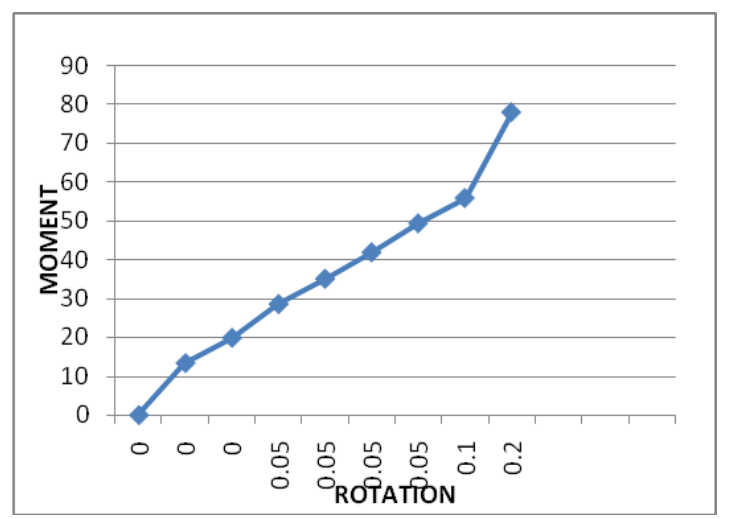

Figure 16 Moment Vs Rotation Normal - 2

FOR SPECIMEN 3:

TABLE 18:Ultimate Moments forNORMAL-3

\begin{tabular}{|c|c|c|c|}
\hline S.NO & LOAD $(\mathrm{kN})$ & M $(\mathrm{kN}-\mathrm{m})$ & ROTATION \\
\hline 1. & 0 & 0 & 0 \\
\hline 2. & 21 & 13.65 & 0.05 \\
\hline 3. & 32 & 20.80 & 0.05 \\
\hline 4. & 42 & 27.30 & 0.05 \\
\hline 5. & 52 & 33.80 & 0.05 \\
\hline 6. & 63 & 40.95 & 0.05 \\
\hline 7. & 73 & 47.45 & 0.10 \\
\hline 8. & 96 & 62.40 & 0.10 \\
\hline
\end{tabular}

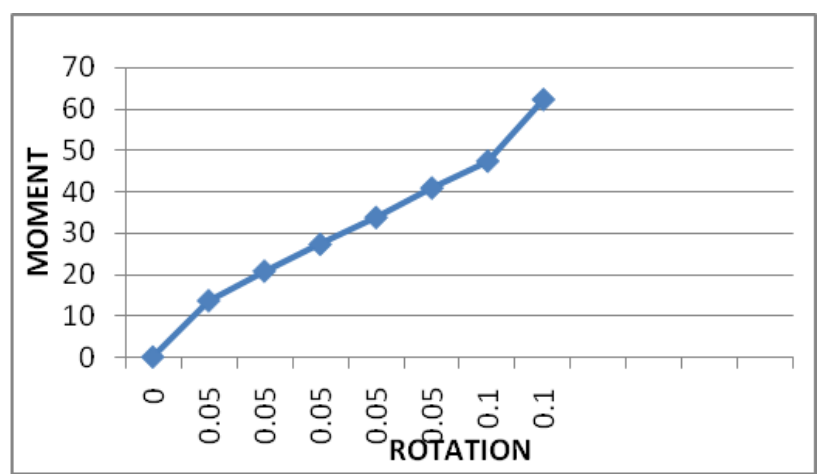

Figure 17 Moment Vs Rotation Normal - 3

\subsection{STIFFNESS}

Stiffness is the rigidity of an object - the extent to which it resists deformation in response to an implemented force.

Stiffness is measured in pressure in keeping with unit period $(\mathrm{N} / \mathrm{mm})$, and is equivalent to the "force steady" in Hooke'S Law.

$$
\text { Stiffness } \mathrm{K}=\boldsymbol{P} / \Delta \boldsymbol{y}
$$

TABLE 19:Initial Stiffness and Final Stiffness

\begin{tabular}{|c|c|c|c|c|}
\hline $\begin{array}{c}\text { S.N } \\
\mathrm{O}\end{array}$ & $\begin{array}{c}\text { SPECIME } \\
\mathrm{N}\end{array}$ & $\begin{array}{c}\text { INITIAL } \\
\text { STIFFNE } \\
\text { SS } \\
(\mathrm{N} / \mathrm{mm}) \\
\end{array}$ & $\begin{array}{c}\text { FINAL } \\
\text { STIFFNE } \\
\text { SS } \\
(\mathrm{N} / \mathrm{mm}) \\
\end{array}$ & $\begin{array}{c}\text { AVERA } \\
\text { GE } \\
(\mathrm{N} / \mathrm{mm})\end{array}$ \\
\hline 1. & HYFRC-1 & 47.5 & 5.397 & \multirow{3}{*}{$\underset{3}{4.569 \times 10}$} \\
\hline 2. & HYFRC-2 & 37.5 & 4.35 & \\
\hline 3. & HYFRC-3 & 5.55 & 3.96 & \\
\hline 4. & $\mathrm{~N}-1$ & 23.53 & 9.575 & \multirow{3}{*}{$2.18 \times 10^{3}$} \\
\hline 5. & $\mathrm{~N}-2$ & 11.44 & 6.818 & \\
\hline 6. & $\mathrm{~N}-3$ & 21 & 4 & \\
\hline
\end{tabular}

\subsection{DUCTILITY}

Ductility isdefined As the potential of a material to deform without difficulty upon the application of tensile force, or because the potential of material to with stand plastic deformation with out rupture.

As no yield point found, no ductility $\mathrm{p}-\Delta$ relation is linear upto failure in HYFRC beams. HYSD

$$
\text { Ductility }=\Delta / \Delta \boldsymbol{y}
$$

TABLE 20:Ductility Result

\begin{tabular}{|c|c|c|}
\hline S.NO & SPECIMEN & DUCTILITY \\
\cline { 1 - 2 } 1. & HYFRC-1 & \multirow{2}{*}{ NO YIELD } \\
\cline { 1 - 2 } 2. & HYFRC-2 & \\
\hline 3. & HYFRC-3 & 0.104 \\
\hline 4. & $\mathrm{~N}-1$ & 0.146 \\
\hline 5. & $\mathrm{~N}-2$ & 0.250 \\
\hline 6. & $\mathrm{~N}-3$ & \\
\hline
\end{tabular}

4.8 ENERGY DISSIPATION CAPACITY:

A reinforced concrete member dissipate energy by experincing in elastic behaviour during cyclic loading. The test result shown below in the table 21 .

\begin{tabular}{|c|c|c|c|}
\hline S.NO & SPECIMEN & $\begin{array}{c}\text { Energy } \\
\text { dissipation } \\
\text { capacity }(\mathrm{N}-\mathrm{mm})\end{array}$ & $\begin{array}{c}\text { AVERAGE } \\
(\mathrm{N}-\mathrm{mm})\end{array}$ \\
\hline 1. & HYFRC-1 & 0.254 & \multirow[t]{3}{*}{0.201} \\
\hline 2. & HYFRC-2 & 0.163 & \\
\hline 3. & HYFRC-3 & 0.187 & \\
\hline 4. & $\mathrm{~N}-1$ & 0.068 & \multirow[t]{3}{*}{0.140} \\
\hline 5. & $\mathrm{~N}-2$ & 0.134 & \\
\hline 6. & $\mathrm{~N}-3$ & 0.226 & \\
\hline
\end{tabular}

TABLE 21:Energy dissipation capacity result

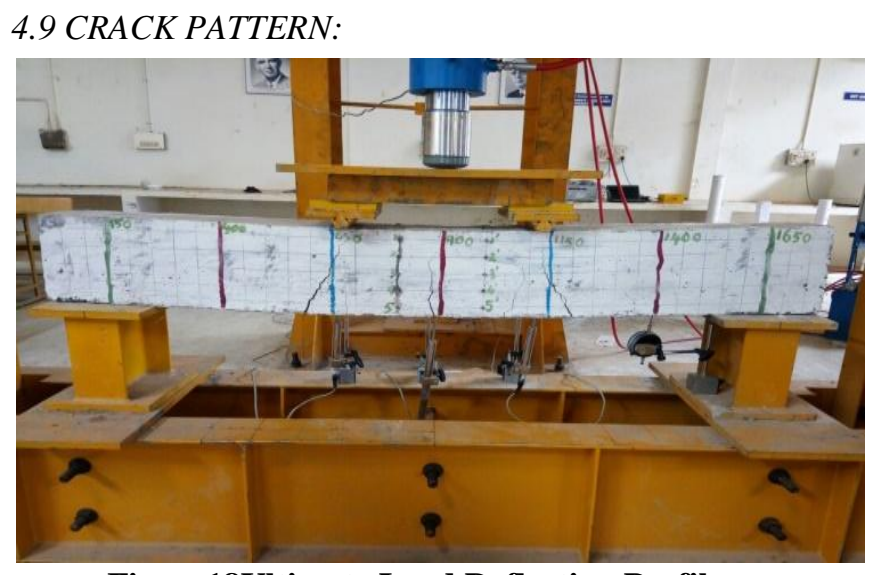

Figure18Ultimate Load Deflection Profile 


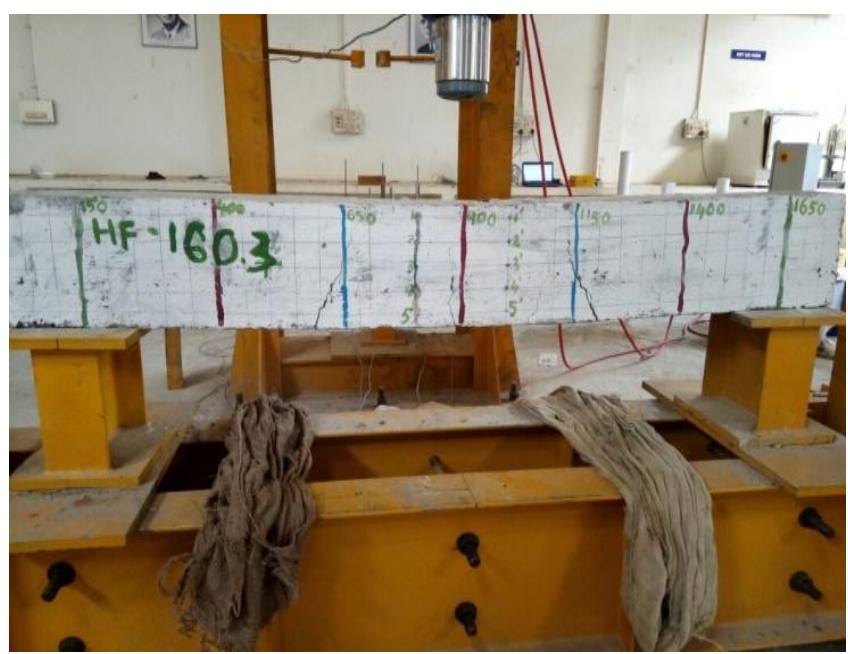

Figure19 After load removal beam regain its normal position

\section{CONCLUSION}

Based at the experimental research conducted on beams under factors loading. The following conclusions are drawn:

$>$ The most compressive cut up tensile and flexural strength HYFRC beam greater than conventional concrete.

> The load sporting capability of HYFRC beam turned into found to be $29 \%$ extra than the price of conventional concrete beam.

$>$ The price of HYFRC beam for load Vs deflection is ready $29.7 \mathrm{mmgreater}$ than conventional concrete beam which is $16.36 \mathrm{~mm}$. GFRP does now not under is going failure. Failure happens in concrete

$>$ The stiffness of HYFRC beam turned into found to be $1.09 \%$ extra than the price of conventional concrete beam.

$>$ As no yield point observed, no ductility p- relation is linear upto failure in HYFRC beams. But conventional concrete beam that's zero. 166 .

$>$ The price of HYFRC beam for power dissipation capacityis about 43.57 greater than traditional concrete beam.

$>$ Replacement of metallic bar with GFRP bar beam has proven better result in flexural load wearing capacities.

$>$ The addition of hybrid fibre at concrete reduces the crack beneath loading conditions. The brittleness of concrete additionally may be advanced with the useful resource of the addition of metallic and glass fibre. Since concrete vulnerable in anxiety, the fibres are beneficial in axial-tension to boom tensile strength.

$>$ The use of GFRP bars in beam has yielded not most effective more flexural electricity to the beam however also properly shear capacities and bending second.

$>$ GFRP bar have weaker elasticity modulus, which generate extra deflection for equal and span.

$>$ The common value of crack initiation masses for beam with GFRP and TMT reinforcement have been $35 \mathrm{KN}$ and $20.06 \mathrm{KN}$ respectively. The average fee of closing load sporting capacity for beams with GFRP and TMT reinforcement have been 100 twenty $5.8 \mathrm{KN}$ and 90 seven.Five $\mathrm{KN}$ respectively.

\section{REFERENCE}

1. Mr.RanjithKumar.R ,Ms.Vennila.A,” Experimental Investigation on Hybrid Fibre Reinforced Concrete", International Journal of Emerging Trends in Engineering and Development, Vol.2 (March 2013), $\mathrm{PP}(39-45)$.

2. Selina ruby G., Geethanjali C., Jaisonvarghese, P. Muthupriya," Influence of Hybrid Fiber on Reinforced Concrete", International Journal of Advanced Structures and Geotechnical Engineering, Vol. 03, Jan 2014,PP(40-43).

3. Kavita S Kene, Vikrant S Vairagade and SatishSathawane, Bonfring, "Experimental Study on Behavior of Steel and Glass Fiber Reinforced Concrete Composites", International Journal of Industrial Engineering and Management Science, Vol. 2, No. 4, December 2012,PP(1-4).

4. P. Sangeetha, "Study On The Compression And Impact Strength Of Gfrc With Combination Of Admixtures", Journal of Engineering Research and Studies, , Vol.2 (JUNE 201),PP(36-40).

5. Wakchaure M. R., Rajebhosale S. H., Satpute M. B., Kandekar S. B, "Comparison Of Compressive Strength And Flexural Shear Strength For Hybrid Fibre Reinforced Concrete With The Controlled Concrete", International Journal of Engineering and Technical Research,Volume-02, September 2014,PP(172-175).

6. G. Suguna B.E, Mrs.S.Parthiban M.E, "Experimental and Investigation of Hybrid Fiber Reinforced Concrete" International Journal of Innovative Science, Engineering \& Technology, Vol. 3, May 2016, PP(409-414).

7. R.H. Mohankar, M.D. Pidurkar, P.V Thakre, S.S. Pakhare, "Hybrid Fibre Reinforced Concrete," International Journal of Science, Engineering and Technology Research, Volume 5, January 2016,(1-4)

8. V. MadhuKiran, Brijbhushan S, Dr.Prakash K B, "A Comparative Study On Mechanical Properties Of Hybrid Fiber Reinforced Concrete With Controlled Concrete", International Research Journal of Engineering and Technology ,Vol: 02 ,Sep2015,PP(402-407).

9. G B. Maranan, A C. Manalo, W Karunasena, B Benmokrane, D Lutze "Flexural behaviour of glass fibre reinforced polymer bars subjected to elevated temperature",23rd Australasian Conference on the Mechanics of Structures and Materials, vol. I, 9 Dec(20014), pp. 187-192

10. Austin Beau Connor "Experimental investigation on the shear characteristics of gfrp reinforcement systems embedded in concrete" Electronic Theses and Dissertations,(2014),pp-1 to 81.

11. Shahul Mohammed, S.Natarajan "Experimental study on flexural behaviour of rc beams strengthened with g.f.r.p" International Journal For Research In Emerging Science And Technology, volume-3, jun2016 ,pp- 1 to 7.

12. Pappula Ravi Kumar,E.BalakoteswarRao "Flexural behaviour of rc beam retrofitted with gfrp", International Journal

\& Magazine of Engineering, Technology, Management

and 
Research A Peer Reviewed Open Access International Journal , Volume No: 2, September 2015

13. Ali S. Shanour, Ali S. Shanour, Maher A. Adam , Mohamed Said "Experimental investigation of concrete beams reinforced with gfrp bars" International Journal Of Civil Engineering And Technology, Volume 5, November (2014), Pp- 279 to 282.

14. Ramadass S \& Job Thomas "Flexure-shear analysis of concrete beam reinforced with gfrp bar", The 5th International Conference on FRP Composites in Civil Engineering, September 2010,pp-1 to 5.

15. S. Marvel Dharma, S. YaminiRoja "Review on behaviour on glass fibre reinforced polymer RC members", International Conference on Explorations and Innovations in Engineering \& Technology (2016), pp-21 to 23.

16. Shrikant M. Harl, "Review on the performance of glass fiber reinforced concrete", International Journal of Civil Engineering Research,Volume 5, (2014), pp. 281-284 